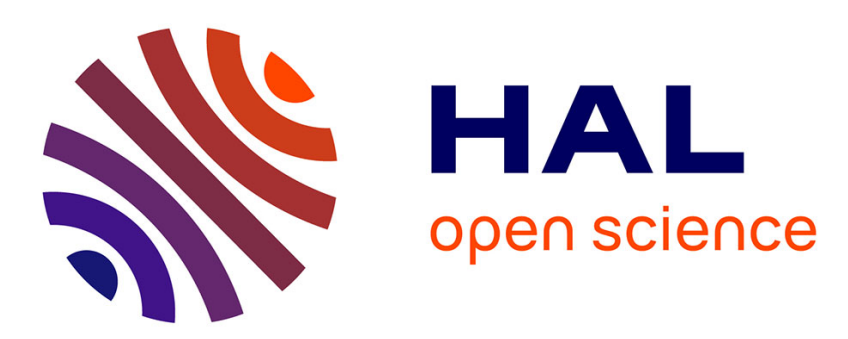

\title{
Online Leader Selection for Collective Tracking and Formation Control: the Second Order Case
}

\author{
Antonio Franchi, Paolo Robuffo Giordano, Giulia Michieletto
}

\section{To cite this version:}

Antonio Franchi, Paolo Robuffo Giordano, Giulia Michieletto. Online Leader Selection for Collective Tracking and Formation Control: the Second Order Case. IEEE Transactions on Control of Network Systems, 2019, 6 (4), pp.1415-1425. 10.1109/TCNS.2019.2891011 . hal-01964754

\author{
HAL Id: hal-01964754 \\ https://hal.laas.fr/hal-01964754
}

Submitted on 23 Dec 2018

HAL is a multi-disciplinary open access archive for the deposit and dissemination of scientific research documents, whether they are published or not. The documents may come from teaching and research institutions in France or abroad, or from public or private research centers.
L'archive ouverte pluridisciplinaire HAL, est destinée au dépôt et à la diffusion de documents scientifiques de niveau recherche, publiés ou non, émanant des établissements d'enseignement et de recherche français ou étrangers, des laboratoires publics ou privés. 


\title{
Online Leader Selection for Collective Tracking and Formation Control: the Second Order Case
}

\author{
Antonio Franchi ${ }^{1}$, Paolo Robuffo Giordano ${ }^{2}$, Giulia Michieletto ${ }^{3}$
}

\begin{abstract}
In this work, we deal with a double control task for a group of interacting agents having a second-order dynamics. Adopting the leader-follower paradigm, the given multi-agent system is required to maintain a desired formation and to collectively track a velocity reference provided by an external source only to a single agent at time, called the 'leader'. We prove that it is possible to optimize the group performance by persistently selecting online the leader among the agents. To do this, we first define a suitable error metric able to capture the tracking performance of the multi-agent group while maintaining a desired formation through a (even time-varying) communication-graph topology. Then we show that this depends on the algebraic connectivity and on the maximum eigenvalue of the Laplacian matrix of a special directed graph depending on the selected leader. By exploiting these theoretical results, we finally design a fully-distributed adaptive procedure able to periodically select online the optimum leader among the neighbors of the current one. The effectiveness of the proposed solution against other possible strategies is confirmed by numerical simulations.
\end{abstract}

\section{INTRODUCTION}

For multi-agent systems, the tracking of a collective motion constitutes a well-studied problem in both the control and agentic communities (see, e.g., the recent [1] but also [2], [3], [4]). Most of the proposed tracking algorithms relies upon the leader-follower paradigm, a very popular technique [5], [6], [7], [8], [9], [10], [11], [12] which envisages the presence of a special agent, referred to as the leader, that has access to the reference motion (often provided by an external source) to be propagated to the whole group. This approach arises as a very powerful tool in real applications, mainly because in many situations it is unfeasible to communicate at the same time with all the agents in the group especially if they are geographically distributed and the available bandwidth is limited.

Within the multi-agent context, the leader-follower solutions have to guarantee the propagation of the reference motion and its tracking with the smallest possible error/delay by means of proper local actions. For this reason, the selection of the leader plays an important role, and the literature distinguishes between static and online leader selection. In the first case, the leader is constantly assumed to be a certain agent within the group chosen at the beginning of the task by the whole multiagent system. Contrarily, when adopting the online selection, the leader is left free to change over time.

${ }^{1}$ LAAS-CNRS, Université de Toulouse, CNRS, Toulouse, France, antonio.franchi@laas.fr

${ }^{2} \mathrm{CNRS}$ at Irisa and Inria Rennes Bretagne Atlantique, Campus de Beaulieu, 35042 Rennes Cedex 4, France. prgeirisa.fr

${ }^{3}$ Dept. of Information Engineering, University of Padova, Italy giulia.michieletto@unipd.it

This research was partially supported by the ANR, Project ANR-17-CE33$0007 \mathrm{MuRoPhen}$
Related works - Both in static and online case, the leader selection generally rests upon the optimization of a suitable index. For example, the authors of [13] have addressed the static leader election task accounting for the maximization of the network coherence, defined as the ability of the consensusnetwork to reject stochastic disturbances, while in [14] the Harmonic Influence Centrality measure is used to quantify the influence of a node on the opinion of the global network. Allowing for the presence of multiple (static) leaders, in [15], a fully distributed strategy is described to select the minimum set of leaders that ensures the structural controllability of the resulting communicating system, whereas a pre-specified number of leaders is assumed in [16] focusing on the computation of bounds on the global optimal value in large stochastically forced consensus networks. Similar scenario is considered in [17] where the K-leader selection problem (standard static leader selection issue) is investigated in ring and path graphs assuming that leaders are noise-free and followers obey noisy consensus dynamics. The authors of [18] instead evaluate the effect of noise-corrupted leaders in the network performance through the definition of the joint centrality of a set of nodes. Finally, in [19] the combinatorial nature of the problem of choosing $k$ leaders among $n$ agents is analyzed showing that the task can be efficiently faced via a semidefinite program, once applied a suitable sequence of relaxations.

Although the literature about online leader selection is more limited, the authors of [20] have addressed this problem by investigating the instantaneous impact of the (time-varying) leaders on the remaining agents through the notion of manipulability. In [21], instead, both the total and the maximum variance of the deviation from a desired trajectory are taken into account to face the so-called in-network leader selection problem designing a self-stabilizing algorithm that, after a topology change, ensures the network stability until the online determination of the optimal leader for the new topology. Such approach rests on agents cooperation: the determination of a distributed control protocol guaranteeing the leadership uniqueness constitutes the main challenge of both static and online leader selection. Within the static context, this issue has been tackled in [22] by using explicit message passing among the formation, while a fault detection strategy without explicit communication need is exploited in [23]. Allowing for a time-varying leadership, the leader identity becomes as an additional degree of freedom that has to be handled over time by the network in a distributed manner limiting the selection duration and its computational burden.

Contributions - Differently from all the aforementioned works in [24] we have proposed to perform the online leader 
selection to simultaneously optimize the collective tracking performance from an external source and the maintenance of a desired formation shape. The goal of this work is to considerably extend the results achieved in [24] by considering a more complex dynamics for the multi-agent group: while in [24] the agents behave as first-order systems, we now consider a second-order dynamics, thus assuming the linear accelerations as control input. The presentation of the contributions follows the same structure of [24] to clearly highlight the differences arising from the adoption of the double-integrator dynamic model. These are clearly stated at the end of this work and mainly derive from the fact that the metrics introduced in [24] are not valid anymore in the second-order case.

For a group of agents modeled as second-order systems, we first formalize the problem of tracking an external reference motion while maintaining a desired formation assuming that the leader identity and the interaction graph topology can be both time-varying. Then, we analyse the effect of a changing leadership for accomplishing the formation control task by showing a direct dependence of the convergence of a suitable defined tracking error on the leader identity. Finally, we propose a new distributed leader election procedure whose effectiveness is validated by means of numerical simulations.

\section{Modeling of Collective External Reference \\ TRACKING AND DESIRED Formation MAINTENANCE}

The first contribution of this work is a set of results regarding the modeling of a multi-agent scenario consisting of a group of $N$ mobile agents equipped with communication, sensing and computation capabilities. Each agent $i$, $i \in\{1 \ldots N\}$, of the group is considered as a point mass in $\mathbb{R}^{d}$, with $d \in\{2,3\}$. The $i$-th agent position is denoted by $\mathbf{p}_{i} \in \mathbb{R}^{d}$ and its linear velocity by $\dot{\mathbf{p}}_{i}=\mathbf{v}_{i} \in \mathbb{R}^{d}$. The set of the linear accelerations $\left\{\dot{\mathbf{v}}_{i}\right\}_{i=1}^{N}$ will be considered as the control input set in the following development.

An undirected graph $\mathcal{G}$, called interaction graph, describes the inter-agent sensing and communication capabilities so that the corresponding adjacency matrix $\mathbf{A} \in\{0,1\}^{N \times N}$ is such that $[\mathbf{A}]_{i j}=1$ if agents $i$ and $j, j \neq i$, can communicate and measure their relative position $\mathbf{p}_{i j}=\mathbf{p}_{i}-\mathbf{p}_{j} \in \mathbb{R}^{d}$, and $[\mathbf{A}]_{i j}=0$ otherwise, $\forall i, j \in\{1 \ldots N\}$. The neighborhood $\mathcal{N}_{i}=\left\{j \mid A_{i j}=1\right\}$ of the node $i$ in $\mathcal{G}$ denotes thus the set of agents with which the $i$-th one can interact. The cardinality of this set represents the degree of the $i$-th agent, which in turns corresponds with the $i$-th element in the main diagonal of $\mathbf{D}=\operatorname{diag}(\mathbf{A} \mathbf{1}) \in \mathbb{R}^{N \times N}$, i.e., in the diagonal matrix associated to the vector $\mathbf{A} \mathbf{1}$ with $\mathbf{1} \in \mathbb{R}^{N}$ representing a column vector of all ones. The degree matrix $\mathbf{D}$ contributes to the definition of the Laplacian matrix $\mathbf{L} \in \mathbb{R}^{N \times N}$ of $\mathcal{G}$, i.e., $\mathbf{L}=\mathbf{D}-\mathbf{A}$. We assume that the second smallest eigenvalue $\lambda_{2}$ of $\mathbf{L}$ (algebraic connectivity of $\mathcal{G}$ ) is positive, or equivalently, that the Laplacian matrix has rank $N-1$. This condition is guaranteed by the existence of at least one communication path (i.e., a sequence of edges) for any pair of agents in the group, so that the graph $\mathcal{G}$ is connected.

In this work, the multi-agent swarm is required to track a collective motion command provided to the group by an external 'entity' (such as another agent, a planner, or a human operator), referred to as the reference source. We assume that this transmits a certain velocity reference $\mathbf{u}_{r} \in \mathbb{R}^{d}$, which is supposed to be a piecewise constant function with period $T_{r}$ (reference command period). In addition, we assume that the current value of $\mathbf{u}_{r}$ is communicated by the reference source to only one agent of the group at a time, called the 'current' leader and denoted with the, possibly time-varying, index $l$.

The connectivity assumption on the interaction network ensures that the reference velocity can be transmitted to the whole group of agents by exploiting a multi-hop propagation algorithm. Without focusing on particular propagation schemes, we consider the following consensus-based strategy

$$
\begin{aligned}
& \hat{\mathbf{u}}_{l}=\mathbf{u}_{r}, \\
& \dot{\hat{\mathbf{u}}}_{i}=-k_{u} \sum_{j \in \mathcal{N}_{i}}\left(\hat{\mathbf{u}}_{i}-\hat{\mathbf{u}}_{j}\right), \quad \forall i \neq l,
\end{aligned}
$$

where $\hat{\mathbf{u}}_{i} \in \mathbb{R}^{d}$ is the estimation of $\mathbf{u}_{r}$ performed by the $i$-th agent, while the positive scalar gain $k_{u}$ tunes the algorithm convergence speed allowing to model both fast or slow propagation technologies, e.g., high-bandwidth LAN networks or ultrasonic underwater communication, respectively. Note that, for the leader, $\hat{\mathbf{u}}_{l}=\mathbf{u}_{r}$ since the reference is directly available.

To compactly rewrite the propagation model (1)-(2), we introduce the 'in-degree' Laplacian matrix $\mathbf{L}_{l} \in \mathbb{R}^{N \times N}$ of the directed graph $\mathcal{G}_{l}$, that is obtained from $\mathcal{G}$ by removing all the ingoing-edges of $l$. In other words, the matrix $\mathbf{L}_{l}$ is derived from $\mathbf{L}$ by zeroing its $l$-th row so that

$$
\mathbf{L}_{l}=\left[\begin{array}{ccc}
\mathbf{M}_{l, 1} & \boldsymbol{\ell}_{l, 1} & \mathbf{M}_{l, 2} \\
\mathbf{0}^{\top} & 0 & \mathbf{0}^{\top} \\
\mathbf{M}_{l, 3} & \boldsymbol{\ell}_{l, 2} & \mathbf{M}_{l, 4}
\end{array}\right],
$$

where $\mathbf{M}_{l, 1}, \mathbf{M}_{l, 2}, \mathbf{M}_{l, 3}, \mathbf{M}_{l, 4}, \ell_{l, 1}, \ell_{l, 2}$, and $\mathbf{0}$ are matrices and column vectors of proper dimensions. By introducing also the matrix $\mathbf{G}_{l}=-\left(\mathbf{L}_{l} \otimes \mathbf{I}_{d}\right) \in \mathbb{R}^{d N \times d N}$ where $\otimes$ denotes the Kronecker product, and the vector $\hat{\mathbf{u}}=\left[\hat{\mathbf{u}}_{1}^{\top} \ldots \hat{\mathbf{u}}_{N}^{\top}\right]^{\top} \in \mathbb{R}^{d N}$, the estimation dynamics (1)-(2) can be compactly rewritten as

$$
\dot{\hat{\mathbf{u}}}=-k_{u}\left(\mathbf{L}_{l} \otimes \mathbf{I}_{d}\right) \hat{\mathbf{u}}=k_{u} \mathbf{G}_{l} \hat{\mathbf{u}} .
$$

As further task, the agents group needs to maintain a desired formation shape defined through the set of constant (absolute) positions $\mathbf{d}=\left[\mathbf{d}_{1}^{\top} \ldots \mathbf{d}_{N}^{\top}\right]^{\top} \in \mathbb{R}^{d N}$. Based on the actuation and sensing properties of the agents, this goal can be accomplished through several control strategies. For sake of model generality, we here consider a classical consensus-like solution applied to second-order agents, namely

$$
\begin{aligned}
\dot{\mathbf{p}}_{i} & =\mathbf{v}_{i}, \\
\dot{\mathbf{v}}_{l} & =b\left(\mathbf{u}_{r}-\mathbf{v}_{l}\right), \\
\dot{\mathbf{v}}_{i} & =b\left(\hat{\mathbf{u}}_{i}-\mathbf{v}_{i}\right)-k_{p} \sum_{j \in \mathcal{N}_{i}}\left(\mathbf{p}_{i j}-\mathbf{d}_{i j}\right), \quad \forall i \neq l,
\end{aligned}
$$

where $\mathbf{d}_{i j}=\mathbf{d}_{i}-\mathbf{d}_{j} \in \mathbb{R}^{d}$ represents the desired relative position between agents $i$ and $j$. The positive scalar gains $b$ and $k_{p}$ determine the velocity tracking performances and the 'stiffness' of the formation control, i.e., how strongly the agents will react to deviations from their desired formation. The convergence towards the desired configuration is guaranteed by the connectivity of the network interaction graph [25]. 
Exploiting the matrix $\mathbf{G}_{l}$ (previously introduced) and the vectors $\mathbf{p}=\left[\mathbf{p}_{1}^{\top} \ldots \mathbf{p}_{N}^{\top}\right]^{\top} \in \mathbb{R}^{d N}, \mathbf{v}=\left[\mathbf{v}_{1}^{\top} \ldots \mathbf{v}_{N}^{\top}\right]^{\top} \in \mathbb{R}^{d N}$, and $\mathbf{d}=\left[\mathbf{d}_{1}^{\top} \ldots \mathbf{d}_{N}^{\top}\right]^{\top} \in \mathbb{R}^{d N}$, the complete agents group dynamics can be shortened as follows

$$
\begin{aligned}
\dot{\mathbf{p}} & =\mathbf{v}, \\
\dot{\mathbf{v}} & =b(\hat{\mathbf{u}}-\mathbf{v})+k_{p} \mathbf{G}_{l}(\mathbf{p}-\mathbf{d}) .
\end{aligned}
$$

Let us then introduce the formation tracking error, the velocity tracking error and the velocity estimation error, namely

$$
\begin{aligned}
& \mathbf{e}_{\mathbf{p}}=\left(\mathbf{p}-\mathbf{1} \otimes \mathbf{p}_{l}\right)-\left(\mathbf{d}-\mathbf{1} \otimes \mathbf{d}_{l}\right), \\
& \mathbf{e}_{\mathbf{v}}=\mathbf{v}-\mathbf{1} \otimes \mathbf{v}_{l}, \\
& \mathbf{e}_{\hat{\mathbf{u}}}=\hat{\mathbf{u}}-\mathbf{1} \otimes \mathbf{u}_{r} .
\end{aligned}
$$

The first one provides a measure of accuracy in tracking and maintaining the desired formation shape encoded in $\mathbf{d}$, while the second and the third ones represent, respectively, the tracking accuracy of the leader velocity $\mathbf{v}_{l}$, and the error in estimation the reference $\mathbf{u}_{r}$.

Using the properties $b(\hat{\mathbf{u}}-\mathbf{v})-\mathbf{1} \otimes \dot{\mathbf{v}}_{l}=b\left(\mathbf{e}_{\hat{\mathbf{u}}}-\mathbf{e}_{\mathbf{v}}\right)$, $\dot{\mathbf{e}}_{\mathbf{p}}=\mathbf{e}_{\mathbf{v}}, \mathbf{G}_{l}(\mathbf{p}-\mathbf{d})=\mathbf{G}_{l} \mathbf{e}_{\mathbf{p}}$, and taking into account (4), and (8)-(9), the dynamics of the overall error $\mathbf{e}=\left[\mathbf{e}_{\mathbf{p}}^{\top} \mathbf{e}_{\mathbf{v}}^{\top} \mathbf{e}_{\hat{\mathbf{u}}}^{\top}\right]^{\top}$ takes the expression

$$
\dot{\mathbf{e}}=\left[\begin{array}{ccc}
\mathbf{0} & \mathbf{I}_{d N} & \mathbf{0} \\
k_{p} \mathbf{G}_{l} & -b \mathbf{I}_{d N} & b \mathbf{I}_{d N} \\
\mathbf{0} & \mathbf{0} & k_{u} \mathbf{G}_{l}
\end{array}\right] \mathbf{e},
$$

where the reference velocity is assumed constant $\left(\dot{\mathbf{u}}_{r}=\mathbf{0}\right)$.

The system 13 presents some interesting properties whose role is fundamental for deriving the main contributions of this work. In this perspective, we first define

$$
\begin{aligned}
\boldsymbol{\ell}_{l} & =\left[\boldsymbol{\ell}_{l, 1}^{\top} \boldsymbol{\ell}_{l, 2}^{\top}\right]^{\top} \in \mathbb{R}^{N-1}, \\
\mathbf{M}_{l} & =\left[\begin{array}{ll}
\mathbf{M}_{l, 1} & \mathbf{M}_{l, 2} \\
\mathbf{M}_{l, 3} & \mathbf{M}_{l, 4}
\end{array}\right] \in \mathbb{R}^{(N-1) \times(N-1)},
\end{aligned}
$$

where $\mathbf{M}_{l}$ is the matrix obtained from $\mathbf{L}_{l}$ by removing its $l$-th row and column. Moreover, we report here the following (known) facts important for the next developments.

Property 1 (Prop. 1 in [24]). If the graph $\mathcal{G}$ is connected, the following properties hold:

1) $\mathbf{L}_{l} \mathbf{1}=\mathbf{0}, \forall l=1 \ldots N$;

2) $\mathbf{M}_{l} \mathbf{1}=\left(\mathbf{1}^{\top} \mathbf{M}_{l}\right)^{\top}=-\boldsymbol{\ell}_{l}$;

3) $\mathbf{M}_{l}$ is symmetric and positive definite;

4) $\sigma\left(\mathbf{L}_{l}\right)=\sigma\left(\mathbf{M}_{l}\right) \cup\{0\}$, where $\sigma(\mathbf{S})$ represents the spectrum of a square matrix $\mathbf{S}$.

A consequence of this properties is that the matrix $\mathbf{L}_{l}$ has $N$ real non negative eigenvalues even though it is not symmetric. Let $\sigma\left(\mathbf{L}_{l}\right)=\left\{\lambda_{i, l}, i=1, \ldots N \mid 0=\lambda_{1, l} \leq \ldots \leq \lambda_{N, l}\right\}$ and $\sigma(\mathbf{L})=\left\{\lambda_{i}, i=1, \ldots N \mid 0=\lambda_{1} \leq \ldots \leq \lambda_{N}\right\}$ be the spectrum of $\mathbf{L}_{l}$ and $\mathbf{L}$, then the following property holds.

Property 2 (Prop. 2 in [24]). For a graph $\mathcal{G}$ and an induced graph $\mathcal{G}_{l}$ it is $\lambda_{i, l} \leq \lambda_{i}$ for all $i=1 \ldots N$.

Prop. 2 descends from the Cauchy interlacing theorem applied to matrices $\mathbf{L}$ and $\mathbf{M}_{l}$ and it implies that, if $\mathcal{G}$ is connected, then both $\lambda_{2}>0$ and $\lambda_{2, l}>0$, where by analogy we denote $\lambda_{2, l}$ as the 'algebraic connectivity' of $\mathcal{G}_{l}$.
Given these premises, we conclude this behavioring section by formally proving the stability of the system (13).

Proposition 1. If graph $\mathcal{G}$ is connected, the system (13) is asymptotically stable for any positive constants $k_{p}, b, k_{u}$. Furthermore, if

$$
b>b_{c}=2 \sqrt{k_{p} \lambda_{N, l}} \quad \text { (critical damping), }
$$

the system evolution has no oscillatory modes, where $\lambda_{N, l}=$ $\max \sigma\left(\mathbf{M}_{l}\right)$, i.e., the largest positive eigenvalue of $\mathbf{L}_{l}$. Finally, the rates of convergence of $\left[\mathbf{e}_{\mathbf{p}}^{\top} \mathbf{e}_{\mathbf{v}}^{\top}\right]^{\top}$ and $\mathbf{e}_{\hat{\mathbf{u}}}$ are dictated by

$$
-\frac{b}{2}+\frac{1}{2} \sqrt{b^{2}-4 k_{p} \lambda_{2, l}} \text { and }-k_{u} \lambda_{2, l},
$$

respectively, where $\lambda_{2, l}=\min \sigma\left(\mathbf{M}_{l}\right)$, i.e., the smallest positive eigenvalue of $\mathbf{L}_{l}$ (algebraic connectivity of $\mathcal{G}_{l}$ ).

Proof. Since $\mathbf{e}_{\mathbf{p}, l}=\dot{\mathbf{e}}_{\mathbf{p}, l}=\mathbf{e}_{\mathbf{v}, l}=\dot{\mathbf{e}}_{\mathbf{v}, l}=\mathbf{e}_{\hat{\mathbf{u}}, l}=\dot{\mathbf{e}}_{\hat{\mathbf{u}}, l}=\mathbf{0}$, the stability of (13) is determined by the real part of the eigenvalues of the $3(N-1) \times 3(N-1)$ matrix

$$
\mathbf{R}=\left[\begin{array}{ccc}
\mathbf{0} & \mathbf{I}_{(N-1)} & \mathbf{0} \\
-k_{p} \mathbf{M}_{l} & -b \mathbf{I}_{(N-1)} & b \mathbf{I}_{(N-1)} \\
\mathbf{0} & \mathbf{0} & -k_{u} \mathbf{M}_{l}
\end{array}\right] \otimes \mathbf{I}_{d},
$$

that is required to be negative definite. Thanks to the properties of the Kronecker product, we can focus on the first matrix composing $\mathbf{R}$. Being a block upper triangular matrix, it is $\sigma(\mathbf{R})=\sigma\left(\mathbf{R}_{11}\right) \cup \sigma\left(\mathbf{R}_{22}\right)$, where

$$
\mathbf{R}_{11}=\left[\begin{array}{cc}
\mathbf{0} & \mathbf{I}_{(N-1)} \\
-k_{p} \mathbf{M}_{l} & -b \mathbf{I}_{(N-1)}
\end{array}\right] \quad \text { and } \quad \mathbf{R}_{22}=-k_{u} \mathbf{M}_{l} .
$$

The spectrum of $\mathbf{R}_{22}$ is clearly $\sigma\left(\mathbf{R}_{22}\right)=-k_{u} \sigma\left(\mathbf{M}_{l}\right)=$ $\left\{-k_{u} \lambda_{2, l}, \ldots,-k_{u} \lambda_{N, l}\right\}$. On the other hand, for any eigenvalue $\mu_{j}, j \in\{1 \ldots 2(N-1)\}$ of $\mathbf{R}_{11}$ it follows that

$$
\mathbf{R}_{11} \mathbf{v}_{j}=\mu_{j} \mathbf{v}_{j}
$$

where $\mathbf{v}_{j}=\left[\begin{array}{ll}\mathbf{v}_{j, 1}^{\top} & \mathbf{v}_{j, 2}^{\top}\end{array}\right]^{\top} \in \mathbb{R}^{2(N-1)}$ is the unit-norm eigenvector of $\mathbf{R}_{11}$ associated to $\mu_{j}$. Consider the matrix $\left(\mathbf{I}_{2} \otimes \boldsymbol{w}_{i}^{\top}\right) \in \mathbb{R}^{2 \times 2(N-1)}$, where $\boldsymbol{w}_{i} \in \mathbb{R}^{(N-1)}$ is the unitnorm eigenvector of $\mathbf{M}_{l}$ associated to the eigenvalue $\lambda_{i, l}$, $i \in\{2 \ldots N\}$. Left-multiplying both sides of 16 with $\left(\mathbf{I}_{2} \otimes \boldsymbol{w}_{i}^{\top}\right)$ and exploiting the symmetry of $\mathbf{M}_{l}$, we obtain

$$
\underbrace{\left[\begin{array}{cc}
0 & 1 \\
-k_{p} \lambda_{i, l} & -b
\end{array}\right]}_{\mathbf{R}_{\lambda_{i, l}}}\left[\begin{array}{l}
\boldsymbol{w}_{i}^{\top} \mathbf{v}_{j, 1} \\
\boldsymbol{w}_{i}^{\top} \mathbf{v}_{j, 2}
\end{array}\right]=\mu_{j}\left[\begin{array}{l}
\boldsymbol{w}_{i}^{\top} \mathbf{v}_{j, 1} \\
\boldsymbol{w}_{i}^{\top} \mathbf{v}_{j, 2}
\end{array}\right] .
$$

Hence $\mu_{j}$ must also be an eigenvalue of the $2 \times 2$ matrix $\mathbf{R}_{\lambda_{i, l}}$ for every $\lambda_{i, l} \in \sigma\left(\mathbf{M}_{l}\right), i=2 \ldots N$. This directly leads to

$$
\begin{aligned}
\mu_{2 i-1} & =-\frac{b}{2}+\frac{1}{2} \sqrt{b^{2}-4 k_{p} \lambda_{i+1, l}}, \\
\mu_{2 i} & =-\frac{b}{2}-\frac{1}{2} \sqrt{b^{2}-4 k_{p} \lambda_{i+1, l}},
\end{aligned}
$$

for $i=1 \ldots N-1$, and then concludes the proof.

Therefore, both the agent velocities $\mathbf{v}$ and the estimation vector $\hat{\mathbf{u}}$ asymptotically converge to the common reference velocity $\mathbf{u}_{r}$, while the agent positions $\mathbf{p}$ converge to the desired shape $\mathbf{1} \otimes \mathbf{p}_{l}+\mathbf{d}-\mathbf{1} \otimes \mathbf{d}_{l}$. Furthermore, the value of $\lambda_{2, l}$ directly affects the convergence rate of the three error vectors $\mathbf{e}_{\mathbf{p}}, \mathbf{e}_{\mathbf{v}}, \mathbf{e}_{\hat{\mathbf{u}}}$ over time. Since, for a given graph topology $\mathcal{G}$, 
$\lambda_{2, l}$ is determined by the identity of the leader in the group, it follows that maximization of $\lambda_{2, l}$ over the possible leaders results in a faster convergence of the tracking error. This insight then motivates the online leader selection strategy detailed in the rest of the paper, where we will show that such maximization is actually only one of the ingredients for obtaining a faster convergence through online leader selection.

\section{Role of LEADER IN TRACKING PERformance}

In this section we provide a theoretical analysis of how the dynamics of the error vector is affected by changing the leader of the agents group at time $t_{k}=k T$ with $k \in \mathbb{N}$ and $T>0$ (leader election period). Since it is reasonable to assume that the internal group communication is much faster than the reference source/leader interaction, we suppose $T \leq T_{r}$ so that the velocity reference $\mathbf{u}_{r}$ remains constant between $t_{k}$ and $t_{k+1}$. Hereafter, we denote the leader at time $t_{k}$ with the index $l_{k}$.

Rewriting the dynamics of the velocity estimation (4) and of the system (8)-(9) among consecutive sampling times, i.e., during the interval $\left[t_{k}, t_{k+1}\right)$, we obtain

$$
\begin{array}{ll}
\dot{\hat{\mathbf{u}}}=k_{u} \mathbf{G}_{l_{k}} \hat{\mathbf{u}}, & t \in\left[t_{k}, t_{k+1}\right), \\
\dot{\mathbf{p}}=\mathbf{v}, & t \in\left[t_{k}, t_{k+1}\right), \\
\dot{\mathbf{v}}=b(\hat{\mathbf{u}}-\mathbf{v})+k_{p} \mathbf{G}_{l_{k}}(\mathbf{p}-\mathbf{d}), & t \in\left[t_{k}, t_{k+1}\right),
\end{array}
$$

with the following initial conditions where $t_{k}^{-}$coincides with the right extreme of the previous time interval 1

$$
\begin{aligned}
& \hat{\mathbf{u}}\left(t_{k}\right)=\hat{\mathbf{u}}\left(t_{k}^{-}\right)+\left(\overline{\mathbf{S}}_{l_{k}} \otimes \mathbf{I}_{d}\right)\left(\mathbf{1} \otimes \mathbf{u}_{r}\left(t_{k}\right)-\hat{\mathbf{u}}\left(t_{k}^{-}\right)\right), \\
& \mathbf{p}\left(t_{k}\right)=\mathbf{p}\left(t_{k}^{-}\right), \\
& \mathbf{v}\left(t_{k}\right)=\mathbf{v}\left(t_{k}^{-}\right) .
\end{aligned}
$$

The matrix $\overline{\mathbf{S}}_{l_{k}} \in \mathbb{R}^{N \times N}$ in 22) realizes the reset action (1) on the components of $\hat{\mathbf{u}}$ related to the new leader $l_{k}$. This is a diagonal matrix having all zeros on the main diagonal except for the $l_{k}$-th entry which is set to one to ensure $\hat{\mathbf{u}}_{l_{k}}\left(t_{k}\right)=\mathbf{u}_{r}\left(t_{k}\right)$. Its complement is defined as $\mathbf{S}_{l_{k}}=\mathbf{I}_{N}-\overline{\mathbf{S}}_{l_{k}}$.

Recalling that $\mathbf{u}_{r}(t)$ is constant in $\left[t_{k}, t_{k+1}\right)$, the dynamics of the error vector $\mathbf{e}(t)=\left[\mathbf{e}_{\mathbf{p}}^{\top}(t) \mathbf{e}_{\mathbf{v}}^{\top}(t) \mathbf{e}_{\hat{\mathbf{u}}}^{\top}(t)\right]^{\top}$ in this interval is correctly described by the system (13). Using (22)-(24), we can derive the initial conditions $\mathbf{e}\left(t_{k}\right)$ as a function of the chosen leader $l_{k}$ and of the received external command $\mathbf{u}_{r}\left(t_{k}\right)$ :

$$
\begin{aligned}
& \mathbf{e}_{\mathbf{p}}\left(t_{k}\right)=\left(\mathbf{S}_{l_{k}} \otimes \mathbf{I}_{d}\right)\left(\left(\mathbf{p}\left(t_{k}^{-}\right)-\mathbf{d}\right)-\mathbf{1} \otimes\left(\mathbf{p}_{l_{k}}\left(t_{k}^{-}\right)-\mathbf{d}_{l_{k}}\right)\right), \\
& \mathbf{e}_{\mathbf{v}}\left(t_{k}\right)=\left(\mathbf{S}_{l_{k}} \otimes \mathbf{I}_{d}\right)\left(\mathbf{v}\left(t_{k}^{-}\right)-\mathbf{1} \otimes \mathbf{v}_{l_{k}}\left(t_{k}^{-}\right)\right), \\
& \mathbf{e}_{\hat{\mathbf{u}}}\left(t_{k}\right)=\left(\mathbf{S}_{l_{k}} \otimes \mathbf{I}_{d}\right)\left(\hat{\mathbf{u}}\left(t_{k}^{-}\right)-\mathbf{1} \otimes \mathbf{u}_{r}\left(t_{k}\right)\right) .
\end{aligned}
$$

From (25,27) it is straightforward to see that the choice of the leader $l_{k}$ directly affects $\mathbf{e}\left(t_{k}\right)$. For this reason, whenever appropriate we will use the notation $\mathbf{e}\left(t_{k}, l_{k}\right)$ to explicitly indicate this (important) dependency.

In order to define a valid metric for the error vector, we first state the following result which holds for any positive semi-definite matrix and then we provide a lemma which is preliminary to the main result of the section.

\footnotetext{
${ }^{1}$ Formally, $t_{k}^{-}$coincides with the one-sided limit of time function approaches $t_{k}$ 'from below'.
}

Proposition 2. Consider the Laplacian matrix $\mathbf{L} \in \mathbb{R}^{N \times N}$ of any connected graph with $N$ vertexes and denote by $\lambda_{N}$ the largest eigenvalue of $\mathbf{L}$. Assuming three constants $k_{n_{1}}, k_{n_{2}}, k_{n_{3}} \in \mathbb{R}$ such that

$$
k_{n_{1}}>0, k_{n_{3}}>0 \text { and } 0<k_{n_{2}}<k_{n_{1}} / \sqrt{\lambda_{N}},
$$

the matrix

$$
\mathbf{P}_{L}:=\left[\begin{array}{ccc}
k_{n_{1}} \mathbf{G}+k_{n_{3}} \mathbf{I}_{d N} & k_{n_{2}} \mathbf{G} & \mathbf{0} \\
k_{n_{2}} \mathbf{G} & k_{n_{1}} \mathbf{I}_{d N} & \mathbf{0} \\
\mathbf{0} & \mathbf{0} & \mathbf{I}_{d N}
\end{array}\right] \in \mathbb{R}^{3 d N \times 3 d N},
$$

where $\mathbf{G}=\left(\mathbf{L} \otimes \mathbf{I}_{d}\right) \in \mathbb{R}^{d N \times d N}$, is positive definite.

Proof. In order to prove the statement it is sufficient to show that the eigenvalues of the symmetric matrix

$$
\mathbf{P}^{\star}=\left[\begin{array}{cc}
k_{n_{1}} \mathbf{L}+k_{n_{3}} \mathbf{I}_{N} & k_{n_{2}} \mathbf{L} \\
k_{n_{2}} \mathbf{L} & k_{n_{1}} \mathbf{I}_{N}
\end{array}\right] \in \mathbb{R}^{2 N \times 2 N},
$$

are all positive. Any eigenvalue $\mu_{j}, j \in\{1 \ldots 2 N\}$ of $\mathbf{P}^{\star}$ must satisfy the relation $\mathbf{P}^{\star} \mathbf{v}_{j}=\mu_{j} \mathbf{v}_{j}$ where $\mathbf{v}_{j} \in \mathbb{R}^{2 N}$ is the eigenvector associated to $\mu_{j}$. If we left-multiply both sides of the previous relation with $\left(\boldsymbol{w}_{i}^{\top} \otimes \mathbf{I}_{2}\right) \in \mathbb{R}^{2 \times 2 N}$, where $\boldsymbol{w}_{i} \in \mathbb{R}^{N}$ is the left-eigenvector of $\mathbf{L}$ associated to the generic eigenvalue $\lambda_{i}, i \in\{1 \ldots N\}$, we obtain

$$
\underbrace{\left[\begin{array}{cc}
k_{n_{1}} \lambda_{i}+k_{n_{3}} & k_{n_{2}} \lambda_{i} \\
k_{n_{2}} \lambda_{i} & k_{n_{1}}
\end{array}\right]}_{\mathbf{P}_{\lambda_{i}}^{\star}}\left[\begin{array}{l}
\boldsymbol{w}_{i}^{\top} \mathbf{v}_{j, 1} \\
\boldsymbol{w}_{i}^{\top} \mathbf{v}_{j, 2}
\end{array}\right]=\mu_{j}\left[\begin{array}{l}
\boldsymbol{w}_{i}^{\top} \mathbf{v}_{j, 1} \\
\boldsymbol{w}_{i}^{\top} \mathbf{v}_{j, 2}
\end{array}\right]
$$

with $\mathbf{v}_{j}=\left[\mathbf{v}_{j, 1}^{\top} \mathbf{v}_{j, 2}^{\top}\right]^{\top}$. This, in turn, implies that $\mu_{j}$ must be an eigenvalue of the 2-by-2 matrix $\mathbf{P}_{\lambda_{i}}^{\star}$ for every $\lambda_{i} \in \sigma(\mathbf{L})$. Analytically computing the eigenvalues of $\mathbf{P}_{\lambda_{i}}^{\star}$ by solving a quadratic equation we obtain

$$
\begin{aligned}
\mu_{j} & =\frac{1}{2}\left(k_{n_{1}}\left(\lambda_{i}+1\right)+k_{n_{3}}-\sqrt{\Delta}\right), \\
\mu_{2 j} & =\frac{1}{2}\left(k_{n_{1}}\left(\lambda_{i}+1\right)+k_{n_{3}}+\sqrt{\Delta}\right),
\end{aligned}
$$

where

$$
\Delta=\left(k_{n_{1}}\left(\lambda_{i}-1\right)+k_{n_{3}}\right)^{2}+4 k_{n_{2}}^{2} \lambda_{i}^{2} .
$$

By then imposing $\mu_{j}, \mu_{2 j}>0$ we obtain

$$
\lambda_{i}^{2} k_{n_{2}}^{2}<k_{n_{1}}^{2} \lambda_{i}+k_{n_{3}} k_{n_{1}}
$$

which is always verified for $\lambda_{i}=0$. For any other $\lambda_{i}>0$, the inequality (35) can be met by adopting the more restrictive constraint $\lambda_{i}^{2} k_{n_{2}}^{2}<k_{n_{1}}^{2} \lambda_{i}$, i.e., $k_{n_{2}}<k_{n_{1}} / \sqrt{\lambda_{i}}$. The proof is concluded by noticing that the last inequality holds for every $\lambda_{i} \in \sigma(\mathbf{L})$ when $k_{n_{2}}$ is set according to 28 .

Lemma 1. Consider a positive definite symmetric matrix $\mathbf{A} \in \mathbb{R}^{N \times N}$ and denote by $0<\phi_{1} \leq \ldots \leq \phi_{N}$ its eigenvalues. Define the symmetric matrix

$$
\begin{aligned}
\mathbf{Q} & :=\operatorname{sym}\left(\mathbf{Q}_{1} \mathbf{Q}_{2}\right)=\frac{1}{2}\left(\mathbf{Q}_{1} \mathbf{Q}_{2}+\mathbf{Q}_{2}^{\top} \mathbf{Q}_{1}^{\top}\right), \text { with } \\
\mathbf{Q}_{1}= & {\left[\begin{array}{ccc}
k_{n_{1}} \mathbf{A}+k_{n_{3}} \mathbf{I}_{M} & k_{n_{2}} \mathbf{A} & \mathbf{0} \\
k_{n_{2}} \mathbf{A} & k_{n_{1}} \mathbf{I}_{M} & \mathbf{0} \\
\mathbf{0} & \mathbf{0} & \mathbf{I}_{M}
\end{array}\right] } \\
\mathbf{Q}_{2}= & {\left[\begin{array}{ccc}
\mathbf{0} & \mathbf{I}_{M} & \mathbf{0} \\
-\mathbf{A} & -b \mathbf{I}_{M} & b \mathbf{I}_{M} \\
\mathbf{0} & \mathbf{0} & -\mathbf{A}
\end{array}\right] }
\end{aligned}
$$


where $b, k_{n_{1}}, k_{n_{2}}, k_{n_{3}}>0$ and also $k_{n_{2}}<k_{n_{1}} / \sqrt{\phi_{N}}$ (to ensure the positive definiteness of $\mathbf{Q}_{1}$, according to Prop. 2). If the following conditions are also met

$$
\begin{aligned}
b & <\phi_{1}, \\
k_{n_{1}} & <\frac{2 \phi_{1}}{b}, \\
k_{n_{2}} & <\min \left\{\frac{b k_{n_{1}}}{\phi_{N}(2+b)}, \frac{2}{b}-\frac{k_{n_{1}}}{\phi_{1}}\right\}, \\
k_{n_{3}} & <\frac{b k_{n_{2}} \phi_{1}}{2},
\end{aligned}
$$

then the $3 N$ eigenvalues of $\mathbf{Q}$ are all negative and in particular they are upper-bounded by the maximum between the following negative quantities

$$
\begin{aligned}
& k_{n_{2}} \phi_{1}\left(b-\phi_{1}\right), \\
& k_{n_{2}} \phi_{N}\left(1+\frac{b}{2}\right)-\frac{b}{2} k_{n_{1}}, \\
& \phi_{1}\left(\frac{b}{2} k_{n_{2}}-1\right)+\frac{b}{2} k_{n_{1}} .
\end{aligned}
$$

Proof. After suitable computations we obtain that

$$
\mathbf{Q}=\left[\begin{array}{ccc}
-k_{n_{2}} \mathbf{A}^{2} & -\frac{b}{2} k_{n_{2}} \mathbf{A}+k_{n_{3}} \mathbf{I}_{M} & \frac{b}{2} k_{n_{2}} \mathbf{A} \\
* & k_{n_{2}} \mathbf{A}-b k_{n_{1}} \mathbf{I}_{M} & \frac{b}{2} k_{n_{1}} \mathbf{I}_{M} \\
* & * & -\mathbf{A}
\end{array}\right]
$$

Any eigenvalue $\nu_{j}, j \in\{1 \ldots 3 N\}$ of $\mathbf{Q}$ must satisfy the relation $\mathbf{Q} \mathbf{v}_{j}=\nu_{j} \mathbf{v}_{j}$ where $\mathbf{v}_{j} \in \mathbb{R}^{3 N}$ is the eigenvector related to $\nu_{j}$. Left-multiplying both sides of the previous relation by $\left(\mathbf{I}_{3} \otimes \boldsymbol{w}_{i}^{\top}\right) \in \mathbb{R}^{3 \times 3 N}$, where $\boldsymbol{w}_{i} \in \mathbb{R}^{N}$ is the left-eigenvector of $\mathbf{A}$ associated to the eigenvalue $\phi_{i}$, $i \in\{1 \ldots N\}$, we get

$$
\left[\begin{array}{ccc}
-k_{n_{2}} \phi_{i}^{2} & -\frac{b}{2} k_{n_{2}} \phi_{i}+k_{n_{3}} & \frac{b}{2} k_{n_{2}} \phi_{i} \\
* & k_{n_{2}} \phi_{i}-b k_{n_{1}} & \frac{b}{2} k_{n_{1}} \\
* & * & -\phi_{i}
\end{array}\right]\left[\begin{array}{l}
\boldsymbol{w}_{i}^{\top} \mathbf{v}_{j, 1} \\
\boldsymbol{w}_{i}^{\top} \mathbf{v}_{j, 2} \\
\boldsymbol{w}_{i}^{\top} \mathbf{v}_{j, 3}
\end{array}\right]=\nu_{j}\left[\begin{array}{c}
\boldsymbol{w}_{i}^{\top} \mathbf{v}_{j, 1} \\
\boldsymbol{w}_{i}^{\top} \mathbf{v}_{j, 2} \\
\boldsymbol{w}_{i}^{\top} \mathbf{v}_{j, 3}
\end{array}\right]
$$

where $\mathbf{v}_{j}=\left[\mathbf{v}_{j, 1}^{\top} \mathbf{v}_{j, 2}^{\top} \mathbf{v}_{j, 3}^{\top}\right]^{\top}$. This means that for every eigenvalue $\phi_{i}$ of $\mathbf{A}, \nu_{j}$ must be an eigenvalue of the $3 \times 3$ matrix

$$
\mathbf{Q}_{\phi_{i}}=\left[\begin{array}{ccc}
-k_{n_{2}} \phi_{i}^{2} & -\frac{b}{2} k_{n_{2}} \phi_{i}+k_{n_{3}} & \frac{b}{2} k_{n_{2}} \phi_{i} \\
* & k_{n_{2}} \phi_{i}-b k_{n_{1}} & \frac{b}{2} k_{n_{1}} \\
* & * & -\phi_{i}
\end{array}\right]
$$

Applying the Gershgorin Circle Theorem we know that every eigenvalue of $\mathbf{Q}_{\phi_{i}}$ is at least in one of the six disks (in the complex plane) centered on the three main diagonal terms of the matrix, and with radius the sum of the magnitudes of the off-diagonal entries in same column or in the same row. Due to the symmetry of $\mathbf{Q}_{\phi_{i}}$ in our case we have only three disks whose largest intersection with real axis are respectively

$$
\begin{aligned}
z_{i, 1} & :=-k_{n_{2}} \phi_{i}^{2}+\left|\frac{b}{2} k_{n_{2}} \phi_{i}-k_{n_{3}}\right|+\left|\frac{b}{2} k_{n_{2}} \phi_{i}\right|, \\
z_{i, 2} & :=k_{n_{2}} \phi_{i}-b k_{n_{1}}+\left|\frac{b}{2} k_{n_{2}} \phi_{i}-k_{n_{3}}\right|+\left|\frac{b}{2} k_{n_{1}}\right|, \\
z_{i, 3} & :=-\phi_{i}+\left|\frac{b}{2} k_{n_{2}} \phi_{i}\right|+\left|\frac{b}{2} k_{n_{1}}\right| .
\end{aligned}
$$

By using the fact that $k_{n_{1}}, k_{n_{2}}, k_{n_{3}}, \phi_{i}$, and $b$ are positive quantities and $k_{n_{3}}$ also satisfies (42) we obtain

$$
\begin{aligned}
& z_{i, 1}<-k_{n_{2}} \phi_{i}^{2}+b k_{n_{2}} \phi_{i}=: \bar{z}_{i, 1}, \\
& z_{i, 2}<k_{n_{2}} \phi_{i}\left(1+\frac{b}{2}\right)-\frac{b}{2} k_{n_{1}}=: \bar{z}_{i, 2}, \\
& z_{i, 3}=-\phi_{i}+\frac{b}{2} k_{n_{2}} \phi_{i}+\frac{b}{2} k_{n_{1}} .
\end{aligned}
$$

Our goal is then to find the additional conditions on $k_{n_{1}}, k_{n_{2}}$ and $k_{n_{3}}$ such that $\bar{z}_{i, 1}, \bar{z}_{i, 2}$ and $z_{i, 3}$ are all negative for each $i \in\{1 \ldots N\}$. Posing $\bar{z}_{i, 1}<0$ results in $b<\phi_{i}$ which is then equivalent to 39. Condition $\bar{z}_{i, 2}<0$ can be guaranteed if $k_{n_{2}}<\frac{b}{\phi_{i}(2+b)} k_{n_{1}}$ which is equivalent to $k_{n_{2}}<\frac{b}{\phi_{N}(2+b)} k_{n_{1}}$. Condition $z_{i, 3}<0$ results in $k_{n_{2}}<\frac{2}{b}-\frac{k_{n_{1}}}{\phi_{i}}$, i.e., $k_{n_{2}}<\frac{2}{b}-\frac{k_{n_{1}}}{\phi_{1}}$. Since it must be also $k_{n_{2}}>0$, the last inequality can only be verified if $\frac{2}{b}-\frac{k_{n_{1}}}{\phi_{1}}>0$, i.e., if 40, holds. Condition 41) is the combination of the just mentioned upper-bounds on $k_{n_{2}}$, namely it has to be simultaneously guaranteed that $k_{n_{2}}<\frac{b}{\phi_{N}(2+b)} k_{n_{1}}$ and $k_{n_{2}}<\frac{2}{b}-\frac{k_{n_{1}}}{\phi_{1}}$. Finally the values in (43), 44), and 45) represent the values of $\bar{z}_{i, 1}, \bar{z}_{i, 2}$, and $z_{i, 3}$, respectively, where $\phi_{i}$ has always been chosen as the worst case according to the asumption that $0<\phi_{1} \leq \ldots \leq \phi_{N}$.

The following result finally gives an explicit characterization of the behavior of $\mathbf{e}(t)$ during the interval $\left[t_{k}, t_{k+1}\right)$ which then naturally leads to the subsequent definition of optimal leader selection. For the sake of exposition, we assume that $k_{p}=k_{u}=1$. All the results easily extend to the more general case $k_{p}>0, k_{u}>0$ with a more tedious machinery.

Proposition 3. Consider the error vector $\mathbf{e}(t)$ with a specific leader $l_{k}$ and, w.l.o.g., $k_{p}=k_{u}=1$. If the constants $b, k_{n_{1}}$, $k_{n_{2}}, k_{n_{3}}$ are chosen so as to satisfy the next conditions

$$
\begin{aligned}
& 0<b<\lambda_{2, l_{k}}, 0<k_{n_{1}}<\frac{2 \lambda_{2, l_{k}}}{b}, 0<k_{n_{3}}<\frac{b k_{n_{2}} \lambda_{2, l_{k}}}{2}, \\
& 0<k_{n_{2}}<\min \left\{\frac{b k_{n_{1}}}{\lambda_{N, l_{k}}(2+b)}, \frac{2}{b}-\frac{k_{n_{1}}}{\lambda_{2, l_{k}}}, \frac{k_{n_{1}}}{\sqrt{\lambda_{N, l_{k}}}}\right\},
\end{aligned}
$$

then the error metric $\|\mathbf{e}(t)\|_{L}^{2}:=\mathbf{e}^{\top}(t) \mathbf{P}_{L} \mathbf{e}(t)$ is monotonically decreasing in the time interval $\left[t_{k}, t_{k+1}\right)$ wherein the topology is assumed to be fixed. In particular, the error metric behavior is dominated by the following exponential upper bound

$$
\|\mathbf{e}(t)\|_{L}^{2} \leq\left\|\mathbf{e}\left(t_{k}\right)\right\|_{L}^{2} e^{-2 \nu_{l_{k}}\left(t-t_{k}\right)} \quad \forall t \in\left[t_{k}, t_{k+1}\right),
$$

where $\nu_{l_{k}}>0$ is the minimum among the following quantities

$$
\nu_{l_{k}}=\min \left\{\begin{array}{l}
k_{n_{2}} \lambda_{2, l}\left(\lambda_{2, l}-b\right), \\
\frac{b}{2} k_{n_{1}}-k_{n_{2}} \lambda_{N, l}\left(1+\frac{b}{2}\right), \\
\lambda_{2, l}-\frac{b}{2}\left(k_{n_{2}} \lambda_{2, l}+k_{n_{1}}\right) .
\end{array}\right.
$$

Proof. The dynamics of the error $\mathbf{e}(t)$ in $\left[t_{k}, t_{k+1}\right)$, with $k_{p}=k_{u}=1$ reduces to

$$
\left[\begin{array}{c}
\dot{\mathbf{e}}_{\mathbf{p}} \\
\dot{\mathbf{e}}_{\mathbf{v}} \\
\dot{\mathbf{e}}_{\hat{\mathbf{u}}}
\end{array}\right]=\left[\begin{array}{ccc}
\mathbf{0} & \mathbf{I}_{d N} & \mathbf{0} \\
\mathbf{G}_{l} & -b \mathbf{I}_{d N} & b \mathbf{I}_{d N} \\
\mathbf{0} & \mathbf{0} & \mathbf{G}_{l}
\end{array}\right]\left[\begin{array}{l}
\mathbf{e}_{\mathbf{p}} \\
\mathbf{e}_{\mathbf{v}} \\
\mathbf{e}_{\hat{\mathbf{u}}}
\end{array}\right]
$$

where we omit (as in the following) the time dependency for the sake of exposition. The sub-vectors $\mathbf{e}_{\mathbf{p}, l}, \mathbf{e}_{\mathbf{v}, l}$, and $\mathbf{e}_{\hat{\mathbf{u}}, l}$ are zero at $t=t_{k}$ and their dynamics is invariant, due to the row of zeros in $\mathbf{L}_{l}$ corresponding to the agent $l$, i.e.,

$\mathbf{e}_{\mathbf{p}, l}=\mathbf{e}_{\mathbf{v}, l}=\mathbf{e}_{\hat{\mathbf{u}}, l}=\dot{\mathbf{e}}_{\mathbf{p}, l}=\dot{\mathbf{e}}_{\mathbf{v}, l}=\dot{\mathbf{e}}_{\hat{\mathbf{u}}, l}=\mathbf{0}, \forall t \in\left[t_{k}, t_{k+1}\right)$.

Hence we can restrict our analysis to the dynamics of the orthogonal subspace, i.e., of the remaining components $\mathbf{e}_{\mathbf{p}, i}$, 
$\mathbf{e}_{\mathbf{v}, i}$, and $\mathbf{e}_{\hat{\mathbf{u}}, i}, \forall i \neq l$. We denote by ${ }^{l} \mathbf{e}_{\mathbf{p}},{ }^{l} \mathbf{e}_{\mathbf{v}}$, and ${ }^{l} \mathbf{e}_{\hat{\mathbf{u}}}$ the $d(N-1)$-vectors obtained by removing the $d$ entries corresponding to $l$ in $\mathbf{e}_{\mathbf{p}}, \mathbf{e}_{\mathbf{v}}$, and $\mathbf{e}_{\hat{\mathbf{u}}}$, respectively, and with ${ }^{l} \mathbf{e}$ their concatenation. Therefore we have

$$
{ }^{l} \dot{\mathbf{e}}=\left[\begin{array}{c}
{ }^{l} \dot{\mathbf{e}}_{\mathbf{p}} \\
{ }^{l} \dot{\mathbf{e}}_{\mathbf{v}} \\
{ }^{{ }^{\mathbf{e}}}
\end{array}\right]=\left[\begin{array}{ccc}
\mathbf{0} & \mathbf{I}_{(N-1) d} & \mathbf{0} \\
{ }^{l} \mathbf{G}_{l} & -b \mathbf{I}_{(N-1) d} & b \mathbf{I}_{(N-1) d} \\
\mathbf{0} & \mathbf{0} & { }^{l} \mathbf{G}_{l}
\end{array}\right]{ }^{l} \mathbf{e}=\mathbf{D}_{l}{ }^{l} \mathbf{e}
$$

where ${ }^{l} \mathbf{G}_{l}=-\left(\mathbf{M}_{l} \otimes \mathbf{I}_{d}\right) \in \mathbb{R}^{(N-1) d \times(N-1) d}$.

We now consider the error metric dynamics. First, note that

$$
\|\mathbf{e}\|_{L}^{2}=\mathbf{e}^{\top} \mathbf{P}_{L} \mathbf{e}={ }^{l} \mathbf{e}^{\top} \mathbf{P}_{M_{l}}{ }^{l} \mathbf{e}=\left\|^{l} \mathbf{e}\right\|_{M_{l}}^{2},
$$

where $\mathbf{P}_{M_{l}} \in \mathbb{R}^{3 d(N-1) \times 3 d(N-1)}$ is defined as $(29)$ with ${ }^{l} \mathbf{G}_{l}$ and $\mathbf{I}_{d(N-1)}$ in place of $\mathbf{G}$ and $\mathbf{I}_{d N}$, respectively. Notice that the positive definiteness of $\mathbf{P}_{L}$ implies that also $\mathbf{P}_{M_{l}}$ is a positive definite matrix for all $l$, thus the metrics $\left\|{ }^{l} \mathbf{e}\right\|_{M_{l}}^{2}$ are well defined for all $l$ according to Prop. 3 . Hence, we have

$$
\begin{aligned}
\frac{d}{d t}\|\mathbf{e}\|_{L}^{2} & =\frac{d}{d t}\left\|^{l} \mathbf{e}\right\|_{M_{l}}^{2} \\
& =2^{l} \mathbf{e}^{\top} \mathbf{P}_{M_{l}}{ }^{l} \dot{\mathbf{e}}=2^{l} \mathbf{e}^{\top} \mathbf{P}_{M_{l}} \mathbf{D}_{l}{ }^{l} \mathbf{e} \\
& =2^{l} \mathbf{e}^{\top} \operatorname{sym}\left(\mathbf{P}_{M_{l}} \mathbf{D}_{l}\right)^{l} \mathbf{e} \leq 2 \mu_{\max , l}\left\|^{l} \mathbf{e}\right\|_{M_{l}}^{2},
\end{aligned}
$$

where $\mu_{\max , l}$ is the largest eigenvalue of the symmetric part of $\mathbf{P}_{M_{l}} \mathbf{D}_{l}$. Accounting for the equality (54, equation 55) implies that for all $t \in\left[t_{k}, t_{k+1}\right)$ it holds that

$$
\|\mathbf{e}(t)\|_{L}^{2} \leq\left\|\mathbf{e}\left(t_{k}\right)\right\|_{L}^{2} e^{2 \mu_{\max , l_{k}}\left(t-t_{k}\right)},
$$

which coincides with 50 proving that $\mu_{\max , l}=-\nu_{l}$, where $\nu_{l}$ is defined as in the proposition.

To this and, first of all we note that, due to the properties of the Kronecker product, the eigenstructure of $\operatorname{sym}\left(\mathbf{P}_{M_{L}} \mathbf{D}_{l}\right)$ is obtained by repeating $d$ times the structure of matrix $\mathbf{Q}$ in (36) by choosing $\mathbf{A}=\mathbf{M}_{l}$. Applying Lemma 1 with $\mathbf{A}=\mathbf{M}_{l}$ and thus $\phi_{1}=\lambda_{2, l}$ and $\phi_{N}=\lambda_{N, l}$ we obtain that, if $k_{n_{1}}, k_{n_{2}}, k_{n_{3}}$ are chosen as in the assumption of the proposition, then $-\nu_{l}$ is the maximum eigenvalue of $\mathbf{Q}$ which results upper-bounded by the maximum of the quantities in Lemma 1 This, in turn, yields a lower-bound for $\nu_{l}$ by the minimum among the quantities in the proposition.

For the reader's convenience, we report in Fig. 1 the values of $\lambda_{2, l}$ vs. $\lambda_{2}$ and of $\lambda_{N, l}$ vs. $\lambda_{N}$ for different leaders $l$ and across different graph topologies.

Thanks to the upper bound (50), at every instant $t=t_{k}$ it is possible to estimate the maximum future decrease of the error vector $\mathbf{e}(t)$ in the interval $\left[t_{k}, t_{k+1}\right)$. By evaluating (50) at $t=t_{k+1}^{-}$, i.e., just before the next leader selection, we obtain

$$
\left\|\mathbf{e}\left(t_{k+1}^{-}\right)\right\|_{L}^{2} \leq\left\|\mathbf{e}\left(t_{k}, l_{k}\right)\right\|_{L}^{2} e^{-2 \nu_{l_{k}} T} .
$$

Note that both $\mathbf{e}\left(t_{k}, l_{k}\right)$ and $\nu_{l_{k}}$ depend on the value of the current leader $l_{k}$. As a consequence, the right-hand side of (57) can be exploited for choosing the leader at time $t_{k}$ in order to maximize the convergence rate of $\mathbf{e}(t)$ during the interval $\left[t_{k}, t_{k+1}\right)$ and therefore improving, at the same time, both the tracking of the reference velocity and of the desired formation.

These observations are gathered in the following Fact.

Fact 1. Consider a $N$-agent system required to accomplish the dual task modeled in Sec. II according to the leader-follower

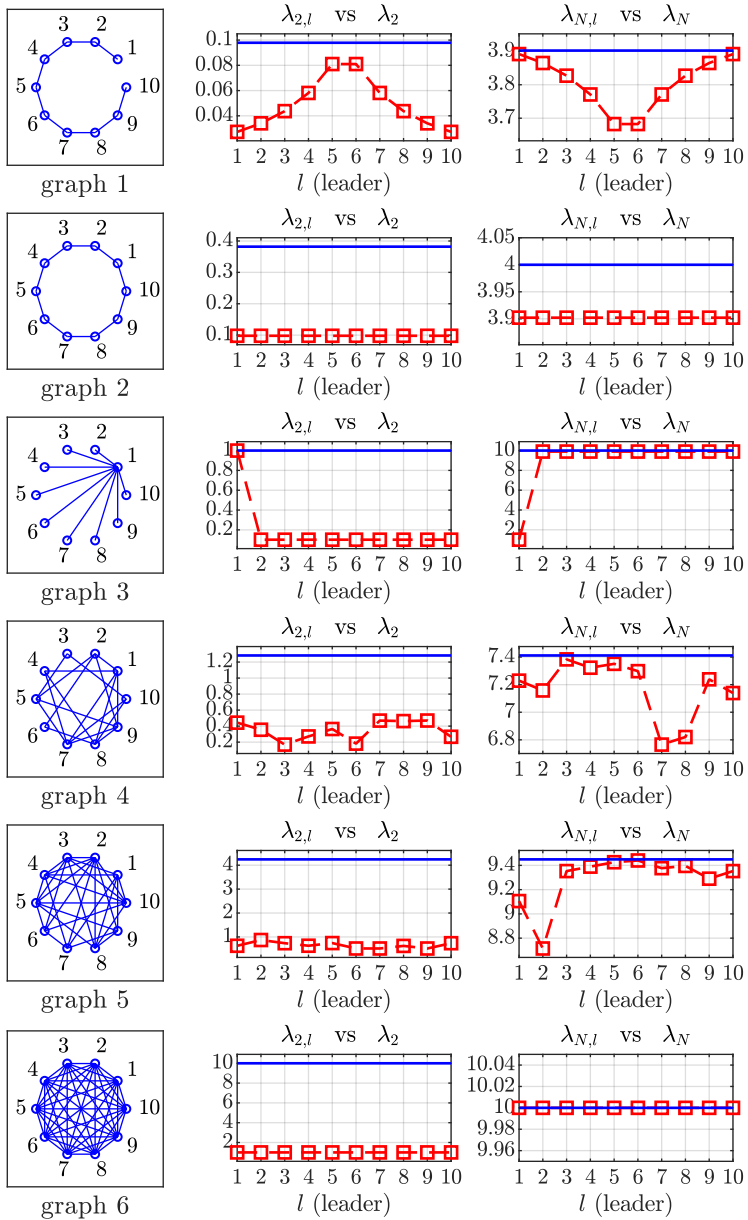

Fig. 1: Values of $\lambda_{2, l}$ vs. $\lambda_{2}$ and $\lambda_{N, l}$ vs. $\lambda_{N}$ for different leaders $l$. The squares correspond to values of $\lambda_{2, l}$ and $\lambda_{N, l}$ associated to a leader $l=1 \ldots N$, with $N=10$. The solid constant blue lines represent $\lambda_{2}$ and $\lambda_{N}$. Each row corresponds to a different graph with $N=10$ vertexes. From the top to the bottom: the line, ring, star, two random (connected) graphs, and a clique graph.

paradigm. Within the online leader selection context, in order to improve the tracking performance of the reference velocity and of the desired formation during the interval $\left[t_{k}, t_{k+1}\right)$ having duration $T$, the leader should be selected so that it solves the following minimization problem

$$
\arg \min _{l \in \mathcal{L}_{k}} f_{k}(l) \text {, with } f_{k}(l)=\left\|\mathbf{e}\left(t_{k}, l_{k}\right)\right\|_{L}^{2} e^{-2 \nu_{l} T},
$$

where $\mathcal{L}_{k} \subseteq\{1 \ldots N\}$ is the set of 'eligible' agents from which a leader can be selected at $t_{k}$ and the error metric defined in Prop. 3 is used.

In Fact 1 the concept of 'eligible' agents is introduced for the first time, however this will be clarified in the next section. In the following, we highlight two crucial aspects related to the minimization problem (58).

Remark 1. Similar to the first-order case, the minimization problem (58) needs to be solved online because of the dependency of the cost function on both the group topology and the current multi-agents system state. 
Remark 2. The reset action (1) implies the zeroing of the components of the estimation error vector $\mathbf{e}_{\hat{u}}$ associated to the current leader $l_{k}$. Hence, the quantity $\left\|\mathbf{e}\left(t_{k}, l_{k}\right)\right\|_{L}^{2}$ may decrease at every instance of the leader selection. Thus, it would be desirable to reduce the leader selection period $T$ as much as possible. However, in practice there exists a maximum frequency at which the leader selection process can be executed guaranteeing the successfully procedure termination under real world constraints. This entails the existence of a finite minimum selection period $T_{\text {min }}$ such that $T \geq T_{\min }>0$.

\section{Distributed Next Best Leader Selection}

The contribution of this section is a fully distributed procedure to solve the optimization problem (58). To cope with the distributiveness requirement, the set of agents eligible as leader, namely the set $\mathcal{L}_{k}$ introduced in Fact 1 is restricted to the neighborhood of the current leader, that is, $\mathcal{L}_{k}=\mathcal{N}_{l_{k}}$. As better explained in the following, this choice ensures the possibility for each agent belonging $\mathcal{L}_{k}$ to evaluate the quantity $f_{k}(l)$ in (58) (depending on the current leader) through local information exchange. Nevertheless, this also implies that the minimization (58) is performed only locally, i.e., in $\mathcal{L}_{k}=\mathcal{N}_{l_{k}}$, thus the achieved minimum might not be global. To guarantee a global optimum, indeed, $f_{k}(l)$ should be minimized over all the agents in the group, i.e., setting $\mathcal{L}_{k}=\{1 \ldots N\}$. However this would result in a fully centralized optimization whenever $\mathcal{G}$ is not the complete graph since it entails that all the agents have a global knowledge of the network. In our method, the global optimum is instead approximated by the repetition, at every time $T$, of the minimization 58 in the neighborhood of the current leader. In this way the computation load is spread among the agents and over time, as customary in distributed approaches. We shall see in Sec. V] that such choice is a good compromise between distributiveness and global optimality.

We now consider the evaluation of the cost function $f_{k}(m)$ in (58) by any leader-candidate $m \in \mathcal{N}_{l_{k}}$. This requires the knowledge of the error norm $\left\|\mathbf{e}\left(t_{k}, m\right)\right\|_{L}^{2}$ and of the eigenvalues $\lambda_{2, m}$ and $\lambda_{N, m}$ in order to compute $\nu_{m}$ through (51). Although all of them are global quantities, in the following we will prove that they can be locally retrieved by restoring on some well-known distributed estimation techniques.

First, for any $m \in \mathcal{N}_{l_{k}}$ both $\lambda_{2, m}$ and $\lambda_{N, m}$ can be locally estimated exploiting a simplified version of the Decentralized Power Iteration algorithm proposed in [26] and based on the PI average consensus estimating (PI-ace) technique introduced in [27]. Thanks to the consensus-based mechanism, the PI-ace strategy allows the $m$-th agent belonging to the group $\mathcal{N}_{l_{k}}$ to build a local estimation $\hat{\boldsymbol{w}}_{k}$ of the eigenvector $\boldsymbol{w}_{k}$ of $\mathbf{M}_{m}$ corresponding to the eigenvalue $\lambda_{k, m}, k \in\{2, N\}$. In this way, the estimate $\hat{\lambda}_{k, m}=-\left(\sum_{n \in \mathcal{N}_{m}}\left[\mathbf{M}_{m}\right]_{m, n}\left[\hat{\boldsymbol{w}}_{k}\right]_{n}\right)\left[\hat{\boldsymbol{w}}_{k}\right]_{m}^{-1}$ can be derived employing only locally available information. The convergence of such a procedure is ensured by a suitable choice of the eigenvector estimation gains and initial conditions as discussed in [26], [27].

Also the error norm $\left\|\mathbf{e}\left(t_{k}, m\right)\right\|_{L}^{2}$ can be estimated by any leader-candidate $m \in \mathcal{N}_{l_{k}}$ via a distributed procedure requiring information locally available and recoverable via 1-hop communication (i.e., directly provided by a neighboring node). To prove this fact, omitting all the dependencies for sake of brevity, we rewrite the scalar quantity $\left\|\mathbf{e}\left(t_{k}, m\right)\right\|_{L}^{2}$ as

$$
\begin{aligned}
\mathbf{e}^{\top} \mathbf{P}_{L} \mathbf{e}=k_{n_{3}} \mathbf{e}_{\mathbf{p}}^{\top} \mathbf{e}_{\mathbf{p}}+k_{n_{1}} \mathbf{e}_{\mathbf{v}}^{\top} \mathbf{e}_{\mathbf{v}}+\mathbf{e}_{\hat{\mathbf{u}}}^{\top} \mathbf{e}_{\hat{\mathbf{u}}}+ & \\
& k_{n_{1}} \mathbf{e}_{\mathbf{p}}^{\top} \mathbf{G} \mathbf{e}_{\mathbf{p}}+2 k_{n_{2}} \mathbf{e}_{\mathbf{p}}^{\top} \mathbf{G e}_{\mathbf{v}},
\end{aligned}
$$

and we note that $\left\|\left(\mathbf{S}_{m} \otimes \mathbf{I}_{d}\right) \boldsymbol{x}\right\|^{2}=\sum_{i=1}^{N}\left\|\boldsymbol{x}_{i}\right\|^{2}-\left\|\boldsymbol{x}_{m}\right\|^{2}$. As a consequence, by recalling (25)-(27) and denoting with the superscript ${ }^{-}$the all quantities computed at $t_{k}^{-}$and with $\tilde{\mathbf{p}}^{-}$the difference $\mathbf{p}^{-}-\mathbf{d}$, the first three terms in 59) can be rewritten accounting for the following identities

$$
\begin{aligned}
\mathbf{e}_{\mathbf{p}}^{\top} \mathbf{e}_{\mathbf{p}} & =\sum_{i=1}^{N}\left\|\tilde{\mathbf{p}}_{i}^{-}-\tilde{\mathbf{p}}_{m}^{-}\right\|^{2}+0= \\
& =\sum_{i=1}^{N} \tilde{\mathbf{p}}_{i}^{-\top} \tilde{\mathbf{p}}_{i}^{-}-2 \tilde{\mathbf{p}}_{m}^{-T} \sum_{i=1}^{N} \tilde{\mathbf{p}}_{i}^{-}+N \tilde{\mathbf{p}}_{m}^{-\top} \tilde{\mathbf{p}}_{m}^{-} \\
\mathbf{e}_{\mathbf{v}}^{\top} \mathbf{e}_{\mathbf{v}} & =\sum_{i=1}^{N}\left\|\mathbf{v}_{i}^{-}-\mathbf{v}_{m}^{-}\right\|^{2}+0 \\
& =\sum_{i=1}^{N} \mathbf{v}_{i}^{-\top} \mathbf{v}_{i}^{-}+2 \mathbf{v}_{m}^{-\top} \sum_{i=1}^{N} \mathbf{v}_{i}^{-}+N \mathbf{v}_{m}^{-\top} \mathbf{v}_{m}^{-} \\
\mathbf{e}_{\hat{\mathbf{u}}}^{\top} \mathbf{e}_{\hat{\mathbf{u}}} & =\sum_{i=1}^{N}\left\|\hat{\mathbf{u}}_{i}^{-}-\mathbf{u}_{r}\right\|^{2}-\left\|\hat{\mathbf{u}}_{m}^{-}-\mathbf{u}_{r}\right\|^{2} \\
& =\sum_{i=1}^{N} \hat{\mathbf{u}}_{i}^{-\top} \hat{\mathbf{u}}_{i}^{-}-2 \mathbf{u}_{r}^{\top} \sum_{i=1}^{N} \hat{\mathbf{u}}_{i}^{-}+N \mathbf{u}_{r}^{\top} \mathbf{u}_{r}-\left\|\hat{\mathbf{u}}_{m}^{-}-\mathbf{u}_{r}\right\|^{2} .
\end{aligned}
$$

Therefore, the quantity $\left\|\mathbf{e}\left(t_{k}, m\right)\right\|_{L}^{2}$ can be evaluated by any agent $m \in \mathcal{N}_{l_{k}}$ as a function of:

1) the vectors $\mathbf{p}_{m}\left(t_{k}^{-}\right), \mathbf{v}_{m}\left(t_{k}^{-}\right)$and $\hat{\mathbf{u}}_{m}\left(t_{k}^{-}\right)$,

2) the vector $\mathbf{u}_{r}\left(t_{k}\right)$,

3) the three vectors $\sum_{i=1}^{N} \hat{\mathbf{u}}_{i}\left(t_{k}^{-}\right), \quad \sum_{i=1}^{N} \mathbf{v}_{i}\left(t_{k}^{-}\right)$, and $\sum_{i=1}^{N}\left(\mathbf{p}_{i}\left(t_{k}^{-}\right)-\mathbf{d}_{i}\right)$

4) the three scalar quantities $\sum_{i=1}^{N} \hat{\mathbf{u}}_{i}^{-\top} \hat{\mathbf{u}}_{i}^{-}, \sum_{i=1}^{N} \mathbf{v}_{i}^{-\top} \mathbf{v}_{i}^{-}$ and $\sum_{i=1}^{N} \tilde{\mathbf{p}}_{i}^{-\top} \tilde{\mathbf{p}}_{i}^{-}$,

5) the total number of agents $N$.

The vectors listed in 1) are locally available to agent $m$ and, similarly, $\mathbf{u}_{r}\left(t_{k}\right)$ is locally available to agent $m$ via 1hop communication from the current leader $l_{k}$. On the other hand, the quantities listed in 3)-4) can be locally estimated employing the PI-ace strategy mentioned before. Finally, the total number of agents $N$ can be assumed to be an a-priori information locally available to each agent, otherwise one can resort to an additional distributed scheme (see, e.g., [28]) to obtain its value over time. This analysis thus proves that any agent $m \in \mathcal{N}_{l_{k}}$ can compute $\left\|\mathbf{e}\left(t_{k}, m\right)\right\|_{L}^{2}$ exploiting only local and 1-hop communication information.

We have thus shown that all the quantities involved in the evaluation of $f_{k}(m)$ in 58 can be locally achieved by any leader-candidate $m \in \mathcal{N}_{k}$. Hence, at every $t_{k}$ the optimal leader selection can be performed by the agents group in a distributed way according to the procedure summarized in Alg. 1. This is hereafter referred as Distributed and Optimized Online Leader Selection (DO2 Leader Selection). Note that its convergence is ensured by the convergence results on the decentralized power iteration method and the PI-ace scheme provided in [26] and [27], respectively.

\section{Simulation Results}

This section is devoted to the validation of the proposed DO2 Leader Selection approach through the comparison with other trivial although intuitive leader selection procedures. These consist of a random leader selection among all the 


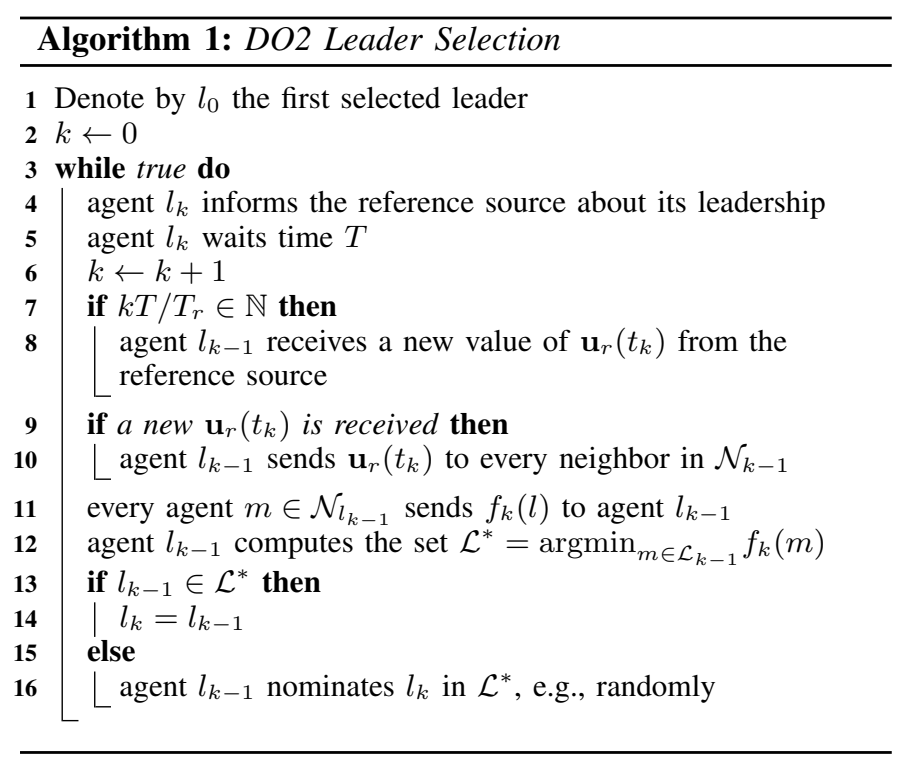

\begin{tabular}{|c|c|}
\hline & leadership changes \\
\hline \hline random leader selection & 288 \\
\hline no leader selection & 0 \\
\hline globally optimized leader selection & 130 \\
\hline locally optimized leader selection & 85 \\
\hline
\end{tabular}

TABLE I: Leadership changes using various leader selections.

agents in the group and a no leader selection envisaging a constant (a priori chosen) leader during all the task execution. To highlight the effectiveness of the DO2 Leader Selection strategy, we also consider its centralized version such that, at each iteration, the leader is selected setting $\mathcal{L}_{k}=\{1 \ldots N\}$ : this allows to evaluate the gap between the global optimal solution and the local one computed through Alg. 1.

Numerical simulations are performed accounting for a group of $N=10$ agents modeled as point masses in $\mathbb{R}^{d}$ with $d=3$. All the runs start from the same initial conditions on the agents position and assume time-varying agents interaction. In detail, the interaction graph $\mathcal{G}$ changes according to Fig. 2 which reports on the top the topology variations with indexing defined in Fig. 1. we simulate the decrease of the connectivity level to show the robustness of the DO2 Leader Selection algorithm w.r.t. to the communication amount. The reference velocity $\mathbf{u}_{r}(t) \in \mathbb{R}^{3}$ is behaviored as a piece-wise constant function of period $T_{r}=5 \mathrm{~s}$ w.r.t. its three components as depicted on the bottom of Fig. 2 For the random and optimized strategies the leader selection period is set to $T=0.05 \mathrm{~s}$. Finally, the gains $k_{p}=2, k_{u}=1, b=0.01$ are used in the network dynamics model and all the PI-ace estimators are designed to converge to the consensus value in a finite number of iterations limiting the final estimation error.

The results of the simulations are shown in Fig. 3 a-d): the plots on the top depict the result of leader selection procedure (i.e., the time-varying leader identity $l(t)$ ) and the plots on the bottom report the corresponding error norm $\left\|\mathbf{e}_{i}(t)\right\|_{L}, i=$ $1 \ldots N$ defined by matrix $\mathbf{P}_{L}$ in 29 highlighting the value related to the current leader (orange line).

First, we can observe that the constant leader strategy

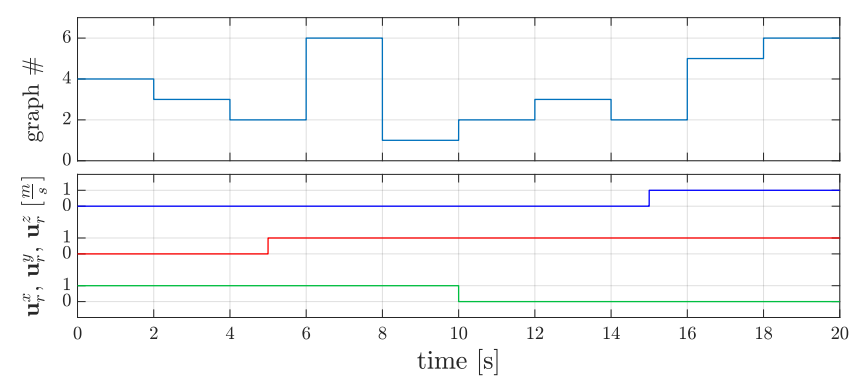

Fig. 2: Current graph $\mathcal{G}$ topology (top) and components of the external velocity reference $\mathbf{u}_{r}$ (bottom).

(Fig. 3p) presents the worst performance in minimizing $\left\|\mathbf{e}_{i}(t)\right\|_{L}$ w.r.t. the other cases, even though the convergence of the errors toward zero is achieved, in accordance with Prop. 3 Note that, w.l.o.g., we have assumed that the leader constantly coincides with the agent 1 . However, when a fixed topology is considered, the performance of the constant leader solution might improve by choosing the leader that optimizes the error convergence rate computing the value $\nu_{l}$ for each $l \in\{1 \ldots 10\}$ according to (51) (see Fig. 1 for the values of $\lambda_{2, l}$ and $\lambda_{N, l}$ ). The random leader selection (Fig. 33) performs better than the constant leader strategy, but its convergence time is much worse w.r.t. the optimized (both globally and locally) leader selection cases in Fig. 3 -d. Indeed, randomly picking the next leader among the neighbors of the current leader makes the error converge to zero in a time between $1 \mathrm{~s}$ and $2 \mathrm{~s}$, while adopting the local DO2 Leader Selection strategy the convergence time is always below $0.5 \mathrm{~s}$. In addition, the results of local DO2 Leader Selection strategy in Fig. 3d concerning the error behavior are comparable to the ones of its global version in Fig. $3 \mathrm{k}$. This implies that the suboptimal solution derived from the use of a distributed paradigm approaches the global optimum provided by the centralized approach. Furthermore, the local DO2 Leader Selection strategy visually results in fewer leader identity changes (plots on the top). To clarify this point, Tab. II reports the number of leadership changes over the considered period for each leader selection strategy.

It has to be noted the following: while the random strategy changes leader almost at each iteration $T$, the DO2 Leader Selection strategy (especially the local version) tends to 'stabilize' the leader choice as the error norm $\left\|\mathbf{e}_{i}(t)\right\|_{L}$ converges to zero, that is when the tracking transient becomes negligible and the group of agents has reached a steady-state in its tracking performance. This fact constitutes an important advantage of the DO2 Leader Selection strategy w.r.t. the random one since in real-world applications the constant change of leadership would correspond to the need of continuously reestablishing new connections from the reference source side. Furthermore, from this point of view to perform a local optimization is more advantageous than a global one.

\section{First-Order vs. SECOND-Order CASE}

In this section, we aim at figuring out the main differences between the first-order leader election addressed in [24] and 


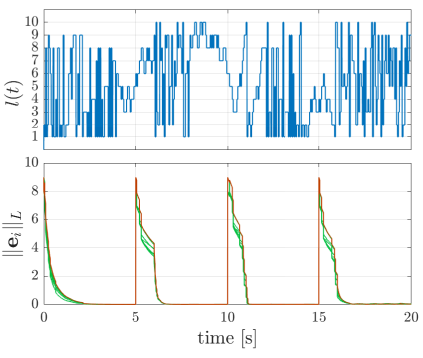

(a) random leader selection

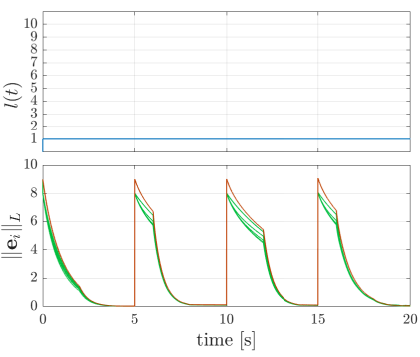

(b) no leader selection
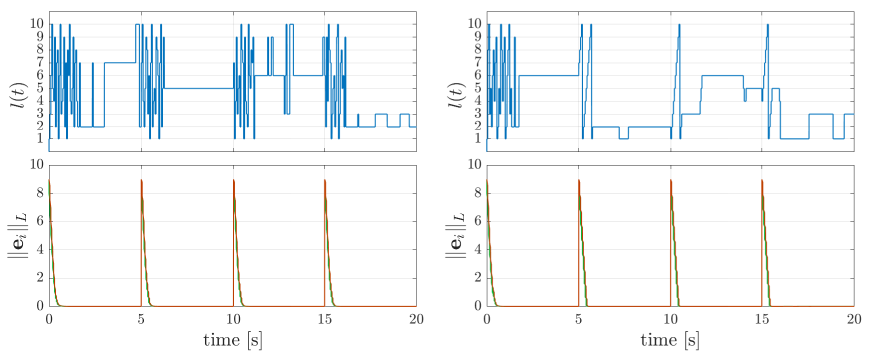

(c) globally optimized leader selection (d) locally optimized leader selection

Fig. 3: Identity of the current leader $l(t)$ (top) and error metric $\left\|\mathbf{e}_{i}(t)\right\|_{L} i=1 \ldots N$ (bottom) applying different leader selection strategies.

the second-order case faced in this work. The purpose is to highlight both the challenging aspects and the benefits deriving from the employment of a more complex dynamic model for the multi-agent group. These are listed in the following.

- In [24] the described agents group is behaviored as a firstorder system since the single-integrator model represents the simplest way to characterize a mobile agent dynamics. Here we deal with second-order systems to describe the dynamics of a multi-agent team. In spite of the increased complexity, this choice is motivated by the fact that these systems better approximate the behavior of physical agentic agents and that controlling acceleration (rather than velocity) generally allows a agent to realize smooth movements.

- Contrary to [24], in general it occurs that $\mathbf{e}_{\mathbf{v}} \neq \mathbf{v}-\mathbf{1} \otimes \mathbf{u}_{r}$. The leader velocity asymptotically converges to the reference $\mathbf{u}_{r}$ via (4), however, since the control acts at acceleration level, one has that $\mathbf{v}_{l} \neq \mathbf{u}_{r}$ during any transient phase.

- The initial condition $\hat{\mathbf{u}}\left(t_{k}\right)$ depends on the chosen leader $l_{k}$ and is in general discontinuous at $t_{k}$. The position vector $\mathbf{p}(t)$ and (contrary to [24]) the velocity vector $\mathbf{v}(t)$ are instead continuous at $t_{k}$.

- Prop. 3 proves that the scalar metric $\|\mathbf{e}(t)\|_{L}^{2}$ is monotonically decreasing along the system trajectories, while this is not guaranteed to hold for other metrics such as the $\ell_{2}$ norm $\|\mathbf{e}(t)\|^{2}$, as well as the one introduced in [24] for the first-order case. Hence, the definition of the matrix $\mathbf{P}_{L}$ and the results stated in Prop. 3 represent novel and original contributions of this work, when comparing with [24].

- $\mathbf{P}_{L}$ is significantly more complex matrix than its firstorder counterpart $\left(\mathbf{P}_{k_{n}}\right.$ in [24] $)$ and it is not at all a straightforward extension of it. $\mathbf{P}_{k_{n}}$ is basically a 'scaled' identity matrix, while $\mathbf{P}_{L}$ contains several repetitions of the Laplacian matrix and is not anymore a diagonal matrix. An important contribution of our work has been to find a matrix, such $\mathbf{P}_{L}$, that 1) can be made positive definite, so that it can represent a well defined norm; 2) makes the matrix $\mathbf{Q}$ positive definite as well, in order to define a monotonically decreasing error dynamics; and 3) has a distributed structure, so that the error can be computed in a distributed way using distributed estimation. Finding such matrix, with a structure completely different from the first- order case, is one of the main cornerstones of this work.

- We have shown that in the first-order leader election, it is required only the knowledge of the smallest eigenvalue $\lambda_{2, m}$ of the matrix $\mathbf{M}_{m}$. Considering a second-order dynamics, it is required also the value of the maximum eigenvalue $\lambda_{N, m}$ since the parameter $\nu_{m}$ depends on both quantities.

- The structure of the Alg. 1 here proposed is similar to the one in [24]. The similarity is however limited since the computation of $f_{k}(m)$ (row 11) in the second-order cases is different from the first-order one. Furthermore, in the second-order case this computation requires the estimation of three (instead of the four as in the first-order case) scalar quantities because of the continuity of the velocity vector v. This results in a simpler implementation compared to first-order case since one less PI-ace filter is needed.

\section{CONCLUSIONS AND Future WORKS}

In this article we deal with the problem of online leader selection for a group of agents whose dynamics is modeled as a second-order system. The key idea is treating the leader identity as a time-varying quantity to be chosen in order to optimize the performance in tracking an external velocity reference signal and in achieving a desired formation shape. For this goal, a suitable tracking error metric has been defined to capture leadership changing effect in the group performance.

A distributed leader selection procedure has then been proposed: during the agent motion, the DO2 Leader Selection algorithm aims at persistently selecting the best leader w.r.t. the defined tracking error metric. The validity of the proposed approach has been stated comparing the DO2 strategy with other more trivial solutions such as keeping a constant leader over time (as typically done), or relying on a random choice.

As future developments we want to extend our analysis allowing the presence of multiple reference sources/leaders and accounting for other optimization criteria such as, e.g., controllability.

\section{REFERENCES}

[1] Y. Wang, E. Garcia, D. Casbeer, and F. Zhang, Cooperative Control of Multi-Agent Systems: Theory and Applications. John Wiley \& Sons, 2017.

[2] R. Olfati-Saber, "Flocking for multi-agent dynamic systems: algorithms and theory," IEEE Trans. on Automatic Control, vol. 51, no. 3, pp. 401420, 2006. 
[3] W. Ren and R. W. Beard, Distributed Consensus in Multi-vehicle Cooperative Control: Theory and Applications. Springer, 2008.

[4] W. Ren and Y. Cao, Distributed Coordination of Multi-agent Networks: Emergent Problems, Models, and Issues. Springer, 2010.

[5] G. Notarstefano, M. Egerstedt, and M. Haque, "Containment in leader-follower networks with switching communication topologies," Automatica, vol. 47, no. 5, pp. 1035-1040, 2011.

[6] Z. Meng, D. V. Dimarogonas, and K. H. Johansson, "Leader-follower coordinated tracking of multiple heterogeneous lagrange systems using continuous control," IEEE Trans. on Robotics, vol. 30, no. 3, pp. 739$745,2014$.

[7] A. Loria, J. Dasdemir, and N. A. Jarquin, "Leader-follower formation and tracking control of mobile robots along straight paths," IEEE Trans. on Control Systems Technology, vol. 24, no. 2, pp. 727-732, 2016.

[8] F. A. Yaghmaie, R. Su, F. L. Lewis, and L. Xie, "Multi-party consensus of linear heterogeneous multi-agent systems," IEEE Trans. on Automatic Control, vol. PP, no. 99, pp. 1-1, 2017.

[9] G. Franzè, W. Lucia, and F. Tedesco, "A distributed model predictive control scheme for leader-follower multi-agent systems," International Journal of Control, pp. 1-14, 2017.

[10] H. Cai and G. Hu, "Distributed tracking control of an interconnected leader-follower multi-agent system," IEEE Trans. on Automatic Control, vol. PP, no. 99, pp. 1-1, 2017.

[11] M. Tognon, C. Gabellieri, L. Pallottino, and A. Franchi, "Aerial comanipulation with cables: The role of internal force for equilibria, stability, and passivity," IEEE Robotics and Automation Letters, Special Issue on Aerial Manipulation, vol. 3, no. 3, pp. 2577 - 2583, 2018.

[12] C. Gabellieri, M. Tognon, L. Palottino, and A. Franchi, "A study on force-based collaboration in flying swarms," in 11th Int. Conf. on Swarm Intelligence, Rome, Italy, Oct. 2018.

[13] S. Patterson and B. Bamieh, "Leader selection for optimal network coherence," in 49th IEEE Conf. on Decision and Control, Atlanta, GA. Dec. 2010, pp. 2692-2697.

[14] L. Vassio, F. Fagnani, P. Frasca, and A. Ozdaglar, "Message passing optimization of harmonic influence centrality," IEEE Trans. on Control of Network Systems, vol. 1, no. 1, pp. 109-120, 2014.

[15] S. Pequito, V. Preciado, and G. J. Pappas, "Distributed leader selection," in 54th IEEE Conf. on Decision and Control, Osaka, Japan, 2015, pp. 962-967.

[16] F. Lin, M. Fardad, and M. R. Jovanović, "Algorithms for leader selection in stochastically forced consensus networks," IEEE Trans. on Automatic Control, vol. 59, no. 7, pp. 1789-1802, 2014.

[17] S. Patterson, "Optimizing coherence in 1-D noisy consensus networks with noise-free leaders," in 2017 American Control Conference, Seattle, WA, May 2017, pp. 3011-3016.

[18] K. Fitch and N. E. Leonard, "Joint centrality distinguishes optimal leaders in noisy networks," IEEE Trans. on Control of Network Systems, vol. 3, no. 4, pp. 366-378, 2016.

[19] M. Fardad, F. Lin, and M. R. Jovanović, "Algorithms for leader selection in large dynamical networks: Noise-free leaders," in 50th IEEE Conf. on Decision and Control and European Control Conference, Orlando,FL, Dec 2011, pp. 7188-7193.

[20] H. Kawashima and M. Egerstedt, "Leader selection via the manipulability of leader-follower networks," in 2012 American Control Conference, Montreal, Canada, Jun. 2012, pp. 6053-6058.

[21] S. Patterson, "In-network leader selection for acyclic graphs," in 2015 American Control Conference, Chicago, IL, Jan 2015, pp. 329-334.

[22] F. Bullo, J. Cortés, and S. Martínez, Distributed Control of Robotic Networks, ser. Applied Mathematics Series. Princeton University Press, 2009.

[23] I. Shames, A. M. H. Teixeira, H. Sandberg, and K. H. Johansson, "Distributed leader selection without direct inter-agent communication," in 2nd IFAC Work. on Estimation and Control of Networked Systems, Annecy, France, Sep. 2010, pp. 221-226.

[24] A. Franchi and P. Robuffo Giordano, "Online leader selection for improved collective tracking and formation maintenance," IEEE Trans. on Control of Network Systems, vol. 5, no. 1, pp. 3-13, 2018.

[25] W. Ren, "Consensus seeking in multi-vehicle systems with a timevarying reference state," in 2007 American Control Conference, New York, NY, Jul. 2007, pp. 717-722.

[26] P. Yang, R. A. Freeman, G. J. Gordon, K. M. Lynch, S. S. Srinivasa, and R. Sukthankar, "Decentralized estimation and control of graph connectivity for mobile sensor networks," Automatica, vol. 46, no. 2, pp. 390-396, 2010

[27] R. A. Freeman, P. Yang, and K. M. Lynch, "Stability and convergence properties of dynamic average consensus estimators," in 45th IEEE Conf. on Decision and Control, San Diego, CA, Jan. 2006, pp. 338-343.
[28] B. Briegel, D. Zelazo, M. Burger, and F. Allgöwer, "On the zeros of consensus networks," in 50th IEEE Conf. on Decision and Control, Orlando, FL, Dec. 2011, pp. 1890-1895.

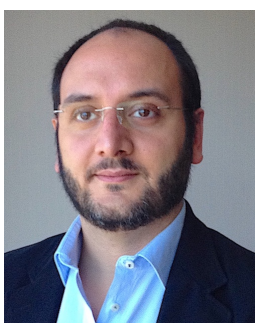

Antonio Franchi (S'07-M'11-SM'16) received the $\mathrm{Ph} . \mathrm{D}$. degree in system engineering from Sapienza University of Rome, Rome, Italy, in 2010. In 2009, he was a Visiting Scholar with University of California at Santa Barbara, Santa Barbara, CA, USA. From 2010 to 2014, he was Research Scientist, Senior Research Scientist, and the Project Leader of the Autonomous Robotics and Human Machine Systems Group, Max Planck Institute for Biological Cybernetics in Tübingen, Germany. Since 2014, he has been a CNRS Researcher with the RIS team, LAAS-CNRS, Toulouse, France. Dr. Franchi is a founding co-chair of the IEEE RAS Technical Committee on Multiple Robot Systems.

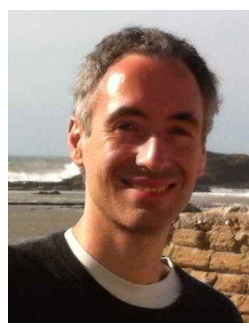

Paolo Robuffo Giordano (M'08-SM'16) received his M.Sc. degree in Computer Science Engineering in 2001, and his Ph.D. degree in Systems Engineering in 2008, both from the University of Rome "La Sapienza". In 2007 and 2008 he spent one year as a PostDoc at the Institute of Robotics and Mechatronics of the German Aerospace Center (DLR), and from 2008 to 2012 he was Senior Research Scientist at the Max Planck Institute for Biological Cybernetics in Tübingen, Germany. He is currently a senior CNRS researcher head of the Rainbow group at Irisa and Inria in Rennes, France.

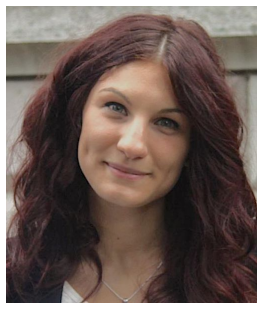

Giulia Michieletto (M'18) received the Master Degree in Automation Engineering and the Ph.D. Degree in Information Engineering from the University of Padova, Italy, in 2014 and 2017 respectively. From March 2016 to February 2017, she was a Visiting Researcher at LAAS-CNRS, Toulouse, France, working in RIS team under the supervision of Antonio Franchi. Since 2018, she is a post-doc fellow with the SPARCS group at University of Padova, Italy. Her main research interests include multi-agent systems modeling and control with a special regard to networked formations of aerial vehicles and nano satellites. 